\title{
The Problem of Overlapping Formation Times
}

\author{
Peter Arnold \\ Department of Physics, University of Virginia, \\ Charlottesville, Virginia 22904-4714, U.S.A \\ E-mail: parnoldevirginia.edu
}

\section{Shahin lqbal*}

National Centre for Physics, Quaid-i-Azam University

Islamabad, Pakistan

E-mail: smi6ndevirginia.edu

\section{Tanner Rase}

Department of Physics, University of Washington,

Seattle, Washington 98195, U.S.A

E-mail: raset@uw. edu

\begin{abstract}
High energy particles such as quarks, gluons, electrons etc. traversing through medium primarily lose energy by showering through hard bremsstrahlung and pair production. These splitting processes are coherent over large distances in the very high energy limit, leading to suppression from the Landau-Pomeranchuk-Migdal (LPM) effect. Avoiding soft-emission approximations, we study the cases where the coherence lengths of two consecutive splittings overlap (which is important for calculating corrections to LPM effect in QCD) and focus on two issues: (i) how to include the effects of non-transverse polarized gauge bosons in the intermediate states, and (ii) how to calculate virtual corrections to in-medium splitting rates, which will be necessary for infrared safe calculations of the characteristics of high energy in-medium parton showers. These in-medium loop calculations require highly non-trivial UV regularization and renormalization. In the current work, we show how to solve these issues for the slightly simpler case of large-Nf QED, where $\mathrm{Nf}$ is the number of electron flavors. Finally, we use these results to calculate corrections to in-medium charge stopping length.
\end{abstract}

International Conference on Hard and Electromagnetic Probes of High-Energy Nuclear Collisions 30 September - 5 October 2018

Aix-Les-Bains, Savoie, France

${ }^{*}$ Speaker. 


\section{Introduction}

Until a millionth of a second after the big bang, the Universe was in a state called the quarkgluon plasma, a hot and dense soup of asymptotically free quarks and gluons. Particle accelerators like LHC and RHIC can recreate conditions similar to the early universe by smashing heavy ions, i.e. nuclei of heavy elements like gold and lead at extremely high energies. These collisions are powerful enough to effectively melt the nucleons in the two colliding nuclei creating for a brief moment, a tiny nucleus-sized fireball of quark gluon plasma. A small fraction of particles in the plasma have exceptionally high energies, and these particles traverse through the plasma, continuously losing energy mainly through the splitting processes of bremsstrahlung and pair production. At high energies, the quantum mechanical "duration" of the splitting process, called the formation time, exceeds the mean free time between collisions with the medium, leading to a significant reduction in the splitting rate. This effect is known as the Landau-Pomeranchuk-Migdal (LPM) effect $[1,2,3]$. A long standing problem in field theory had been to account for the LPM effect in cases where the formation times of two subsequent splittings overlap. Along with Chan [4, 5, 6, 7], we have been working to calculate such overlap corrections to in-medium splitting rates. In the present article, we summarize our recent calculations of overlapping in-medium (i) virtual corrections, (ii) effects of longitudinal polarizations of intermediate state gauge bosons, and (iii) overlap corrections to in-medium energy and charge stopping distances. We briefly describe the physics of the LPM effect below.

\subsection{Landau-Pomeranchuk-Migdal (LPM) Effect}

Analyzing shower development gets significantly complicated because of the LPM effect. To qualitatively understand the LPM effect, consider an electron that scatters in the medium and subsequently radiates a bremsstrahlung photon. The radiated photon cannot resolve details that are smaller than its wavelength. This creates an uncertainty (depicted as the blue shaded region) about the exact time and place of the splitting process. The extent of this uncertainty is called the for-

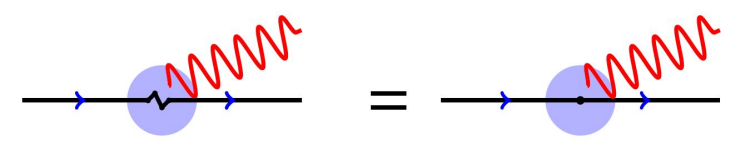

Figure 1: Bremsstrahlung from an electron scattering in the medium. The diagram on the left depicts bremsstrahlung from a series of scatterings with the medium which becomes indistinguishable from bremsstrahlung from a single scattering as shown in the diagram on the right.

mation time, which is roughly speaking, the quantum mechanical duration of the splitting process. When the mean free path of the electron is smaller than the wavelength of the bremsstrahlung photon, bremsstrahlung from multiple small angle scatterings becomes indistinguishable from bremsstrahlung from a single large angle scattering. The observed splitting rate is therefore smaller than the naive expectation and this suppression is known as the Landau-Pomeranchuk-Migdal (LPM) effect which was figured out in the 1950's and its QCD generalization was developed in the 1990's. 


\subsection{Overlapping Formation Times}

Can subsequent splitting processes be treated as if they were independent? Consider for now the case of roughly democratic splitting, where the energies of the two daughters in each splitting process are not too different from each other. In general, the probability of splitting within a formation time is of the order of the coupling $\alpha$ associated with the splitting vertex. Then, the mean free time between splittings is given by $t_{\text {rad }} \sim \frac{t_{\text {form }}}{\alpha}$. Therefore, up to leading order in $\alpha$, a high

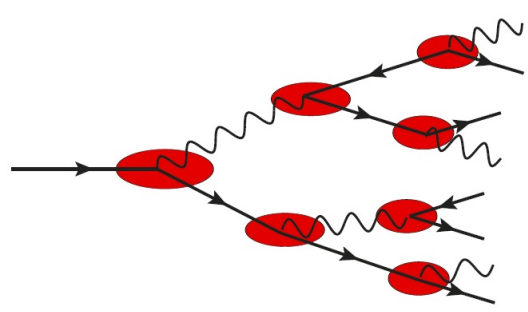

(a)

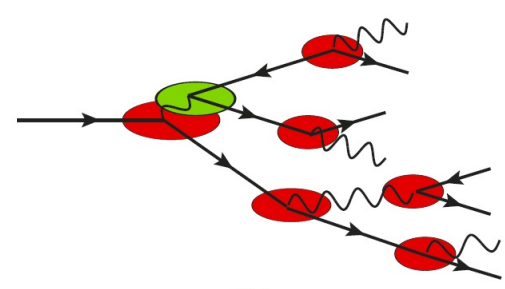

(b)

Figure 2: (a) Non-overlapping splittings that may be treated as quantum mechanically independent. (b) A shower with overlapping splittings that cannot be treated as quantum mechanically independent.

energy in-medium shower can be thought of as a series of non-overlapping mutually independent splittings as shown in Fig. 2(a) above. (The ovals represent the formation lengths associated with each splitting.) However, for energies that are actually reached in heavy ion collision experiments at LHC and RHIC, the value of the coupling is only moderately small. Therefore, splittings with overlapping formation times, as shown in Fig. 2(b) above, could be important. We will focus on calculating effects of such overlapping splittings on in-medium energy and charge stopping distances.

\section{Overview of the Calculation}

\subsection{Previous Work}

Let $x, y$ be the energy fractions of two consecutively radiated particles. Understanding LPM effect for cases of splittings with overlapping formation times had been a long standing problem in field theory. Previous authors $[10,11]$ had considered this problem in the limiting case of $y \ll$ $x \ll 1$. Together with Chang, we subsequently developed field theory formalism to calculate the correction due to overlapping formation times for general values of $x$ and $y$ and used it to calculate the overlap corrections for real, double gluon bremsstrahlung in large-Nc QCD, where $\mathrm{Nc}$ is the number of colors.

\subsection{The Missing Pieces}

Our work so far in this regard was incomplete for two reasons. First, the calculations of Refs. [4, 5, 6, 7] had only considered transversely polarized gluons. However the polarization of intermediate state gauge bosons does not have to be transverse and a full calculation must include the effects of longitudinal polarizations as well. Second, a calculation of in-medium double 
bremsstrahlung alone is not enough to compute medium effects on energy loss and shower development and for that purpose, virtual corrections to single bremsstrahlung must also be calculated.

\subsection{What we calculate}

Here we solve both of these issues, — calculating overlap effects from longitudinal polarizations of intermediate state gauge bosons and calculating in-medium loop corrections to single splitting rate- for the slightly simpler case of large-Nf QED. Where $\mathrm{Nf}$ is the number of electron flavors. In terms of Feynman diagrams, the LPM effect represents quantum mechanical interference between splitting amplitudes from different scattering centers. It turns out, in calculation of LPM effect, it is convenient to think of such interference terms as if they were a single process, as shown in Fig. 3 below. Here, in the figure on the right, the upper blue part represents the amplitude
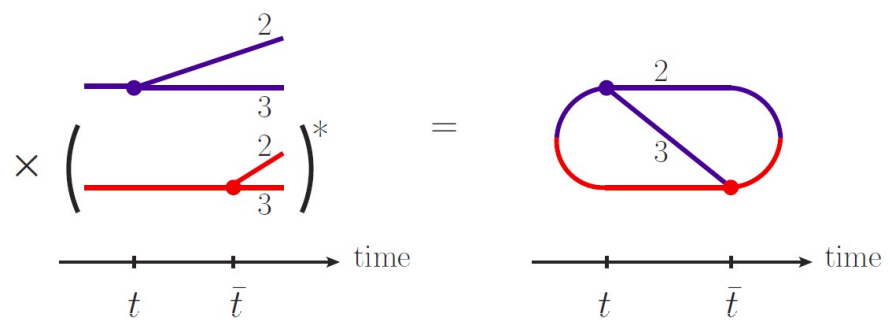

Figure 3: Schematic interference diagram for a leading order single splitting process.

and the lower red represents the conjugate amplitude part of the interference. In this language, the above diagram can be interpreted as a 3-particle evolution sandwiched between splitting vertices at times $t$ and $\bar{t}$. Similarly, the next-to-leading order contributions we need to calculate are also interferences of multiple emission amplitudes at slightly different times.

\subsection{Longitudinal polarizations and Light-Cone Perturbation Theory}

Longitudinal polarizations of intermediate state gauge bosons can be integrated out using Light-Cone Perturbation Theory (LCPT). Integrating out the longitudinal polarizations gives rise to the effective 4-fermion instantaneous vertices as shown below. Here, the bar across the inter-

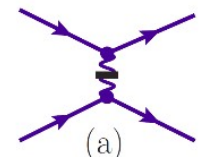

(a)

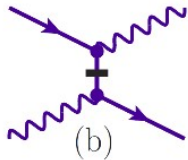

(b)

Figure 4: Effective instantaneous interactions that arise in QED when using Light-Cone Perturbation theory.

mediate line indicates an instantaneous interaction. Including these instantaneous vertices gives additional interference contributions to real double splitting shown in Fig. 5.

\subsection{In-medium loop diagrams}

We find that in-medium virtual processes like the one shown below, turn out to require highly non-trivial regularization and renormalization. However, in case of large- Nf QED, the situation is 

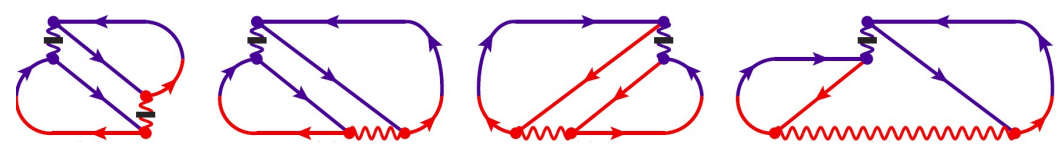

Figure 5: Set of interference contributions to real double splitting involving the effective instantaneous interactions in Large-Nf QED.

simplified slightly. For homogeneous and static medium, the rate for photon bremsstrahlung $e \rightarrow e \gamma$ is independent of the number of electron flavors Nf. However, the probability for pair production $\gamma \rightarrow e \bar{e}$ is proportional to Nf. Therefore, for Large-Nf QED, only the following type of overlap corrections dominate:

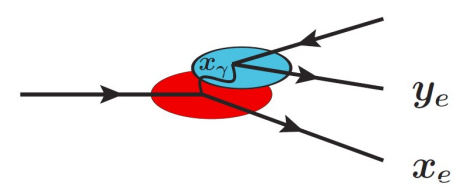

Figure 6: The dominant type of overalp diagrams in Large-Nf QED.

This reduces the number of virtual diagrams that need to be calculated to the seven interference contributions shown in Fig. 7: Note: Fig. 7 is the same as Fig. 8 in Ref. [8]. For making contact
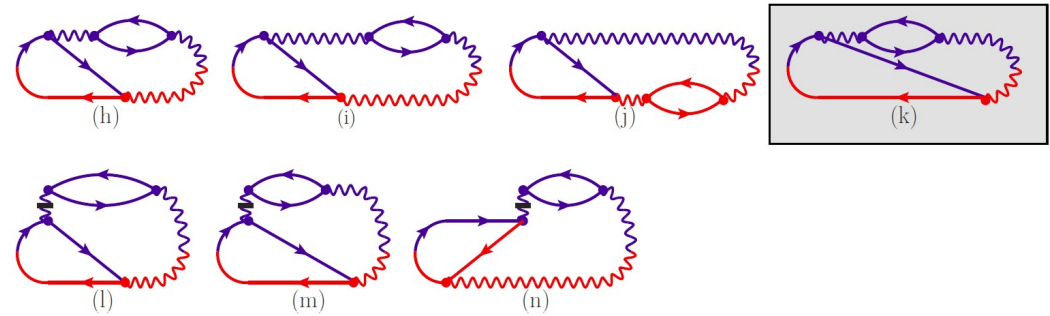

Figure 7: Set of time ordered virtual diagrams that contribute to $e \rightarrow e \gamma$ rate.

with the more detailed discussions in Ref. [8], we have kept diagram labels unchanged. The diagram (k) above, turns out to be the most important, as well as the most difficult one to calculate. However, we find that its non-canceling UV divergence can be regulated and absorbed into the usual renormalization of the QED coupling constant.

\section{Results}

\subsection{Next-to-leading order corrections to splitting rates}

We find the total NLO contribution to the differential splitting rate from all $e \rightarrow e \gamma$ diagrams with overlapping formation times in large-Nf QED is given by

$$
\left[\frac{d \Gamma}{d x_{e}}\right]^{N L O}=-\frac{N_{f} \alpha_{E M}}{6 \pi}\left[\frac{d \Gamma}{d x_{e}}\right]^{L O} \log \left(\frac{x_{e} \mu^{4}}{\left(1-x_{e}\right)^{3} \hat{q} E}\right)+(\text { Stuff }) .
$$


Where "Stuff" includes complicated integrals which we solve numerically, $\mu$ is the renormalization scale and $\hat{q}$ is the transverse momentum squared per unit time that a high energy particle picks up as result of collisions in the medium.

\subsection{Corrections to charge stopping length}

Using these results, we calculate the effects of overlapping formation times on electron charge stopping length. Choosing the renormalization scale $\mu=(\hat{q} E)^{1 / 4}$, we find the relative correction to electron stopping distance $l_{\text {stop }}$ is,

$$
\frac{\Delta l_{\text {stop }}}{l_{\text {stop }}}=-1.302 N_{f} \alpha\left[(\hat{q} E)^{1 / 4}\right]
$$

Where $\Delta l_{\text {stop }}$ is the NLO correction and $l_{\text {stop }}^{0}$ is the leading order result. This shows that unlike the heuristic attempts of accounting for overlap effects, the overall effect of overlapping formation times is to enhance energy loss and reduce the stopping distance. For more detailed discussions, we would refer the readers to our most recent publications $[8,9]$.

\section{References}

[1] L.D. Landau and I. Pomeranchuk, Limits of applicability of the theory of bremsstrahlung electrons and pair production at high-energies (in Russian), Dokl. Akad. Nauk Ser. Fiz. 92 (1953) 535.

[2] L.D. Landau and I. Pomeranchuk, Electron cascade process at very high energies (in Russian), Dokl. Akad. Nauk Ser. Fiz. 92 (1953) 735.

[3] A.B. Migdal, Bremsstrahlung and pair production in condensed media at high-energies, Phys. Rev. 103 (1956) 1811.

[4] P. Arnold and S. Iqbal, "The LPM effect in sequential bremsstrahlung", JHEP 04 (2015) 070 [arXiv:1501.04964 [hep-ph]].

[5] P. Arnold, H.-C. Chang and S. Iqbal, "The LPM effect in sequential bremsstrahlung 2: factorization", JHEP 09 (2016) 078 [arXiv:1605.07624[hep-ph]].

[6] P. Arnold, H.-C. Chang and S. Iqbal, "The LPM effect in sequential bremsstrahlung: dimensional regularization", JHEP 10 (2016) 100 [arXiv:1606.08853[hep-ph]].

[7] P. Arnold, H.C. Chang and S. Iqbal "The LPM effect in sequential bremsstrahlung: 4-gluon vertices", JHEP 10 (2016) 124 [arXiv:1608.05718[hep-ph]].

[8] P. Arnold and S. Iqbal "In-medium loop corrections and longitudinally polarized gauge bosons in high energy showers", [arXiv:1806.08796 [hep-ph]]. JHEP, to appear.

[9] P. Arnold, S. Iqbal and Tanner Rase "Strong-vs. weak coupling pictures of jet quenching: A dry run using QED” [arxiv: 1810.06578[hep-ph]]

[10] B. Wu, "Radiative energy loss and radiative p-broadening of high-energy partons in QCD matter," JHEP 1412, 081 (2014) [arXiv:1408.5459 [hep-ph]].

[11] J. P. Blaizot and Y. Mehtar-Tani, "Renormalization of the jet-quenching parameter," Nucl.Phys. A 929, 202 (2014) [arXiv:1403.2323 [hep-ph]]. 